\title{
Methanol expression regulator 1 (Mxr1p) promotes xylulose 5-phosphate recycle via increaseing transketolase activity in Pichia pastoris
}

\section{Chunjun Zhan}

Jiangnan University https://orcid.org/0000-0001-9504-6439

\section{Yingyue Pan}

Jiangnan University

Xiuxia Liu

Jiangnan University

Chunli Liu

Jiangnan University

Jinling Zhan

Jiangnan University

Dinghua Xu

Sinosbio Biomedical Technologies

\section{Zhonghu Bai}

Jiangnan University

Yankun Yang ( $\nabla$ yangyankun@jiangnan.edu.cn )

Jiangnan University https://orcid.org/0000-0003-4815-6832

\section{Research}

Keywords: Mxr1p, Tkl1p, interaction, Pichia pastoris, MUT regulation, xylulokinase

Posted Date: February 13th, 2020

DOI: https://doi.org/10.21203/rs.2.23470/v1

License: (c) (1) This work is licensed under a Creative Commons Attribution 4.0 International License.

Read Full License 


\section{Abstract}

Background Methanol expression regulator $1(\mathrm{Mxr} 1 \mathrm{p})$ is a key transcription factor that plays a vital role in the methanol utilization pathway in Pichia pastoris ( P. pastoris ). Most genes referred to the methanol utilization pathway were regulated by Mxr1p. However, some genes did not show a significant difference between methanol and glycerol even though they play an important role in the methanol utilization pathway. So far, the regulation mechanism about these genes and the relationship with Mxr1p are still unknown.

Results Methanol metabolic pathway analysis revealed that most of the methanol-induced genes were upregulated in transcriptional level when cultured in methanol. Whereas some genes like tkl1 (transketolase 1) did not show significant up-regulation in methanol even though it plays a very important role in Xu5P recycle, the reason is still not clear. To clarify this point, firstly, pull-down and MS experiments were performed. The result shows that Tkl1p protein combined with Mxr1p in vitro. Subsequently, this result was further confirmed by yeast two-hybrid in vivo, and the binding region mainly located from 150AA to 400AA. Moreover, Ser215 phosphorylation did not affect this interaction. In addition, Mxr1p400AA integration into $\Delta \mathrm{mxr} 1$ could rescue cell growth in methanol. All the above results proved that Mxr1p played a post-translational role in the methanol utilization pathway and Mxr1p-400AA may achieved most of Mxr1p functions. Secondly, the function of Mxr1p-Tkl1p complex was expounded by detecting formaldehyde consumption and xylulose production in cell-free systems. Results showed that Mxr1p-Tkl1p mixture could promote formaldehyde consumption and xylulose production in vitro .

Conclusion Mxr1p promotes methanol utilization via combining with Tkl1p to accelerate Xu5P recycle and this interaction was not affected by Ser 215 phosphorylation.

\section{Background}

As an important heterologous protein expression system, more than 1,000 proteins have been successfully expressed via P. pastoris, including angiostatin, a-glucosidase [1]and Anti-CEACAM5 nanobody [2, 3]. Moreover, as the rare methylotrophic microbes, detailed methanol metabolic pathway especially important intermediates recycle pathway not only significantly promotes other methylotrophic microbes construction but also promotes autotrophic microorganism design [4]. Many results showed that the robust alcohol oxidase 1 promoter $\left(P_{A X_{1} 1}\right)$ contributes most to this successful expression system [5]. Data showed that methanol expression regulation 1 protein (Mxr1p) plays a key role in aox 1 and other methanol-induced genes transcription [6]. For example, aox 1 was repressed in Dmxr1 mutant even though cells were cultured in methanol $[7,8]$. The reason is that Mxr1p binds to methanol-induced promoter e.g., aox 1 , das and pex $8[7,9]$ to promote transcription. Our previous results also revealed that Mxr1p promotes aox 1 transcription via binding on $P_{G T 1}$ to inhibit $g t 1$ transcription $[10,11]$. All of the above results proved that Mxr1p could regulate methanol metabolic pathway by transcription level. Recently, Parua et al. demonstrated that a 14-3-3 protein (GenBank accession no. CCA38880) regulates Mxr1p activity in a carbon source-dependent manner. The mechanism is that 14-3-3 protein binds on Mxr1p 
between residues 212-225. Ser215 phosphorylation promotes the interaction between Mxr1p and the 143-3 protein and subsequently leads to methanol metabolic pathway repression $[12,13]$. However, although phosphorylation of Ser215 is similar in methanol and glucose, cells showed a significant difference in methanol utilization ability. Thus, there may be other proteins interact with Mxr1p to regulate MUT pathway. Based on that, several transcription factors such as Trm1, Prm1 and Mit1 have been identified [14-17]. Disappointedly, there is no interaction among these transcription factors even though some of them, e.g., Mxr1 and Rop1, shared the same binding site in methanol-induced genes[18].

In this report, methanol-induced genes were identified by methanol metabolic pathway analysis based on transcription level. Furthermore, potential proteins (e.g., Tkl1p) interacting with Mxr1p in vitro were identified via pull-down analysis and Mass spectrometry (MS). Yeast two-hybrid screening showed that Tkl1p interacted with Mxr1p in vivo, the interaction region mainly located between 150AA to 400AA and Ser215 phosphorylation did not affect this interaction. Finally, formaldehyde consumption and xylulose production assay proved that the complex formed by Tkl1p and Mxr1p could promote methanol utilization by accelerating xylulose recycle. All of the above results not only be benefit for clarifying Xu5P recycle route but also would help enrich Mxr1p regulation system.

\section{Results}

\section{Transcription level referred to methanol utilization pathway in $P$. pastoris}

\section{Fig. 1.}

Transcriptional levels referred to MUT genes in different media (methanol and glycerol) were detected after 48h. Hierarchical clustering analysis provides a holistic view about MUT genes expression level in methanol or glycerol media (Fig. 1). Results showed that compared with glycerol, aox 1 and das $1 / 2$ were significantly upregulated in methanol. Moreover, das2 upregulation was more significant than das1, which indicated that the regulation mechanism between das 1 and das 2 might be different [19]. As the key intermediate, Xu5P plays a crucial role in formaldehyde fixation and degradation, which means genes related to Xu5P recycle could be promoted in methanol. Results showed that most genes referred to Xu5P recycle route, such as rep 1 and $t a l$, were upregulated in methanol. Surprisingly, $t k / 1$ gene did not show significantly improved as other MUT genes, even though it plays a very important role in Xu5P recycle route (Fig.1).

\section{Detection $t k / 1$ expression levels in different cells}

\section{Fig. 2}

According to transcriptome analysis, many genes related to MUT pathway and non-oxidative pentose phosphate pathway (NOP) were up-regulated in methanol medium. However, $t k / 1$ did not show significant up-regulated like das even though it plays a key role in the carbon rearrangement process, which indicates that maybe some other unrevealed regulation pathways participate in $t k / 1$ regulation. To clarify this 
hypothesis, firstly, the transcriptional and translational level of $t k / 1$ were measured. Results showed that tk/1 did not show a significant difference between methanol and glycerol medium (Fig.2a). It has been reported that many genes were promoted by Mxr1p in MUT and NOP pathway[9], which inspired us that Mxr1p may promote $t k / 1$ expression. To verify this hypothesis, $t k / 1$ expression level and methanol consumption in Dmxr1 mutant and mxr1 over-expression strains were detected. Unfortunately, results showed that Mxr1p did not show obvious improvement for $t k / 1$ both in RNA and protein level (Fig.2b) even though it promotes methanol utilization (Fig.2a).

\section{Excavation Mxr1p-interaction protein via pull-down assay and Yeast two-hybrid assay}

\section{Fig. 3}

The above results indicated that although Tkl1p participates in Xu5P recycle, its transcriptional and translational level did not improve a lot in methanol, which indicated that Tkl1p might be involved in posttranscriptional modification. Based on that, M1 and M2 proteins were purified and pull-down assay was performed in this research to find some candidate proteins (Fig.3a,b), which may interact with Mxr1p in vitro. Results proved that some proteins could combine with Mxr1p in vitro (Fig.3). Each candidate band was collected and sent for MS analysis, results showed the interesting protein is Tkl1p. This result was further confirmed in vivo by yeast two-hybrid assay (Fig.3b). Moreover, we found the diploid containing $\mathrm{M} 1$ and Tkl1p did not grow on selection plate, whereas the diploid containing M2 and Tkl1p grew on selection plate, this result indicated that the region Mxr1p interacting with Tkl1p mainly located between 150AA to 400AA. Our previous results showed that M1 plays a crucial role in the process of binding on $\mathrm{P}_{\mathrm{GT} 1}$ and $\mathrm{P}_{\mathrm{AOX} 1}$ [10]. Some other researchers showed that $\mathrm{N} 400$ of Mxr1p play the main function between protein interaction $[12,13]$. Considering all these results, Mxr1p was divided into two parts; the first part contains a DNA binding domain (mainly $1-150 \mathrm{AA}$ ), which mainly regulates gene transcription. The second is protein interaction domain (mainly 150-400AA), which may be involved in different metabolic pathways via interacting with different proteins.

\section{Tkl1p interact with Mxr1p did not via phosphorylation of Ser215}

\section{Fig. 4}

It has been proved that 14-3-3 protein interacted with Mxr1p via phosphorylation of Ser215. In order to examine whether Ser215 phosphorylation would affect the interaction between M2 and Tkl1p. M2-Ser215 was mutant to M2-Asp215 (M3). Tkl1p-His and M3-Flag protein were purified and used for pull-down assay. The targeted band was recognized by Western blotting (Fig.4a), which indicates that phosphorylation of Ser215 or not did not affect the interaction between Tkl1p and Mxr1p. Meanwhile, different strains growth curve were measured in methanol medium (Fig.4b). Results showed that $m x r 1$ deletion (Dmxr1) affect cell grew. Moreover, when M2 or M3 was integrated back into genome respectively, cell grew would be rescued. This indicated that Mxr1p N-400AA take main function of Mxr1p. 


\section{Functions of the Mxr1-TKL1 complex}

\section{Fig. 5}

Based on Fig.1, it could be inferred that Tkl1p mainly promotes Xu5P recycle in the carbon rearrangement route. Considering all results, one hypothesis is that the complex formed by Mxr1p and Tkl1p may participate in Xu5P recycle route. In order to test this hypothesis, xylulose production was measured in vitro using crude total protein. Results (Fig.5) showed that xylulose production in $t k / 1$ overexpression strain $(32.7 \mathrm{mM}, 1 \%$ formaldehyde $)$ is much higher $(P<0.01)$ than that in wild type $(12.6 \mathrm{mM}, 1 \%$ formaldehyde), which confirmed that Tkl1 p promote Xu5P production. Moreover, when the purified M2 protein was added into this reaction system, higher production of xylulose ( $36.2 \mathrm{mM}, 1 \%$ formaldehyde) was detected $(P=0.0009<0.01)$, the above results proved that the complex formed by Mxr1p and Tkl1p promotes xylulose production. In order to further confirm this result, formaldehyde consumption in different reaction systems were detected, results showed that (Fig.5a) more formaldehyde consumption was detected in Tkl1p-Mxr1p reaction system than others. Based on the above results, it concluded that Mxr1p could combine with Tkl1p to promote Xu5P recycle and further accelerate formaldehyde consumption.

Many intermediates, especially formaldehyde, in methanol utilization are toxic for cells, therefore, how to convert these toxic intermediates into useable substrates is very important for cell to survive. It has been proved that high efficient $\mathrm{Xu} 5 \mathrm{P}$ recycle route would promote formaldehyde degradation and rescue cells[10]. However, no definite results illustrate Xu5P recycle route in methylotrophic microbes. Based on the above results, it showed that complex formed by Mxr1p and Tkl1p participate in Xu5P recycle route. It has been proved that xylulose production increased in vitro experiments. The reason may be that excessive Xu5P was converted into xylulose by some enzyme. To verify this hypothesis, xylulokinase (XK) expression level was measured in vivo, the result showed that XK expression level was promoted in Tkl1Mxr1 over-expression cells, meanwhile, methanol consumption was also improved (Fig.5b).

\section{Discussion}

As an important renewable carbon source, methanol has drawn lots of attention during the past few years due to its low energy cost, liquid form [20-22] and other advantages. Moreover, compared with other one-carbon sources like $\mathrm{CO}_{2}$, methanol could be high efficiency used by native methylotrophic microbes[23]. Besides, both $\mathrm{CO}_{2}$ fixation pathway and formaldehyde degradation pathway share similar intermediate such as Xu5P and Ru5P [24, 25]. Based on the above reasons, lots of attention has been paid to engineer non-native methylotrophic microbes. Unfortunately, most of the recombinant cells did not grow well in methanol. Many reasons may lead to this problem, but inefficiency intermediates supplement may be an important reason. Therefore, Clarify intermediates recycle regulation mechanism in methanol utilization pathway maybe not only beneficial for other non-native methylotrophic microbes construction but also would promote other one-carbon source utilization [4, 26]. In Saccharomyces cerevisiae, Tkl1 has been shown as the rate-limiting factor in NOP pathway [27]. Moreover, in P. pastoris 
Tkl1p showed similar conserved domain structure with Das1p and Das2p. Some results indicated that cells losing das 1 and das 2 are still able to grow in methanol [28], which indicated that Tkl1p may be involved in some other pathways. However, Tkl1 did not showed significant difference between methanol and glycerol as DAS1/2, which also indicated that $t k / 1$ and das $1 / 2$ regulation mechanism might be different [29] .

In this paper, our results showed that Tkl1p interacted with $\mathrm{M} 2$, and the interaction region mainly located between 150AA to 400AA. In addition, the function of Tkl1p-Mxr1p was confirmed to promote Xu5P recycle. However, when we detected XK transcriptional level in different cells, we found that there is no significant difference between Tkl1p overexpression strain and Tkl1p-Mxr1p double overexpression strains (Fig.4), the reason may be that Xu5P could be highly recycled in vivo to support formaldehyde fixation. As a critical transcription factor, Mxr1p is inextricably linked with the methanol utilization pathway both in transcriptional and post-translational [8], which indicated that Mxr1p may be the core of methanol regulation system in $P$. pastoris. In this study, many efforts have been tried to purify integral Mxr1p protein, unfortunately, all of them were failed (Fig.S1/2). Some researchers have indicated that overexpressed Mxr1p is toxic to cells [30], but the reason is not clear now. Our results also indicated that Mxr1p overexpression would significantly inhibit cell growth (Fig.S4). Based on our results, it inferred that Mxr1p might be involved in many different metabolic pathways besides MUT pathway. Therefore, Mxr1p overexpression may inhibit other important metabolic pathways, not 'toxic' for cells.

\section{Conclusion}

In this paper, Tkl1p regulation mechanism was clarified via different experiments, results showed that Tkl1p could bind on Mxr1p specific region (mainly located from 150AA to 400AA), and the complex would promote Xu5P recycle then further promote methanol utilization.

\section{Materials And Methods}

\section{Medium, strain and plasmid}

P. pastoris was cultured in YPD broth and BMY medium along with its derivatives, BMMY, BMGY, BMGMY and BMMGY. Saccharomyces cerevisiae (S. cerevisiae) was incubated in YPD broth and YPDA medium, $\mathrm{Y}_{2} \mathrm{HGold}$ in SD/Try (TAKARA) and Y187 in SD/Leu medium (TAKARA). Constructed plasmids were transformed into Escherichia coli (E. coli) DH5a and Trans110 (Trans Gen) cells, and these cells were cultured at $37^{\circ} \mathrm{C}$ in Luria-Bertani (LB) medium. All formulas used are listed below. YPD (1\% yeast extract, $2 \%$ tryptone, $2 \%$ glucose), BMY (1 L: $10 \mathrm{~g}$ yeast extract, $20 \mathrm{~g}$ tryptone, $3 \mathrm{~g} \mathrm{~K}_{2} \mathrm{HPO}_{4}, 11.8 \mathrm{~g} \mathrm{KH}_{2} \mathrm{PO}_{4}, 13.4 \mathrm{~g}$ $\mathrm{YNB}, 4 \times 10^{-4} \mathrm{~g}$ biotin, $10 \mathrm{~mL}$ glycerol), BMMY (1 L: $10 \mathrm{~g}$ yeast extract, $20 \mathrm{~g}$ tryptone, $3 \mathrm{~g} \mathrm{~K}_{2} \mathrm{HPO}_{4}, 11.8 \mathrm{~g}$ $\mathrm{KH}_{2} \mathrm{PO}_{4}, 13.4 \mathrm{~g} \mathrm{YNB}, 4 \times 10^{-4} \mathrm{~g}$ biotin, $5 \mathrm{~mL}$ methanol), BMGMY (BMGY plus $0.5 \%$ methanol) and BMMGY medium (BMGY plus 0.5\% methanol). YPDA (YPD with the addition of $15 \mathrm{~mL} 0.2 \%$ adenine), LB $\left(0.5 \%\right.$ yeast extract, $1 \%$ tryptone, $1 \% \mathrm{NaCl}$ ), TB/SB $\left(2.6 \mathrm{~g}\right.$ peptone, $4.8 \mathrm{~g}$ yeast extract, $0.462 \mathrm{~g} \mathrm{KH}_{2} \mathrm{PO}_{4}$, 
$2.51 \mathrm{~g} \mathrm{~K}_{2} \mathrm{HPO}_{4}, 2 \mathrm{~g}$ glycerol). SD/Try and SD/Leu medium was purchased from TAKARA. The solid medium was prepared by adding $2 \%$ agar powder.

Electroporation was used to transform P. pastoris. Transformation and recombinant DNA operations were performed as described previously[11]. Selection of marker-resistant colonies was performed using LB with $50 \mu \mathrm{g} / \mathrm{mL}$ ampicillin or kanamycin, and YPD with $0.3 \mathrm{mg} / \mathrm{mL} \mathrm{G} 418$ or $0.1 \mathrm{mg} / \mathrm{mL}$ zeocin.

\section{Strains, plasmids and primers}

See Tables 1, 2 and 3.

\section{Plasmid and strain construction}

\section{M1/2 expression plasmid and strain construction}

Initially, the $P$. pastoris genome was extracted. The sequences of Mxr1-150AA (M1) and Mxr1-400AA (M2) were amplified by PCR using Pfu DNA polymerase (Thermo Scientific, Waltham, MA, USA) and the $P$. pastoris genome. These two sequences vary in the number of $\mathrm{N}$-terminal residues: 150 amino acids (M1) and 400 amino acids (M2), respectively. Subsequently, the PCR products were digested with $\mathrm{Ndel} / \mathrm{Xhol}$, and the digested fragments were inserted into the PSVT7 plasmid. The recombinant plasmid was chemically transformed into $E$. coli $B L 21(D E 3)$ cells.

\section{M1/2-pGBKT7 recombinant plasmid construction}

M1/2 were amplified with Pfu DNA polymerase and the $P$. pastoris genome was used as the template. The Ndel/ Xhol-digested PCR products were ligated into the pGBKT7 plasmid. Recombinant plasmids M1pGBKT7 and M2-pGBKT7 were transformed into $S$. cerevisiae following the protocol provided by TAKARA.

\section{AD-pGADT7 recombinant plasmid construction}

The general procedure was the same as above. Target genes (like $t k / 1$ ) were amplification and the PCR products were double-digested with the same restriction enzymes, and the digested fragments were ligated into the appropriate plasmid. Recombinant plasmid AD-pGADT7 was transformed into $S$. cerevisiae using the protocol provided by TAKARA.

\section{Gene expression studies in E. coli BL21 (DE3) cells}

E. coli BL21(DE3) cells were inoculated into LB medium and grown at $30^{\circ} \mathrm{C}, 230 \mathrm{rpm}$ for $16 \mathrm{~h}$. The cells were then transferred to TB/SB and cultured at $30^{\circ} \mathrm{C}, 230 \mathrm{rpm}$ for $5 \mathrm{~h}$. IPTG at a final concentration of 0.5 $\mathrm{mmol} / \mathrm{L}$ was added to the culture to induce protein expression. Cells were harvested $20 \mathrm{~h}$ later by centrifugation at $4{ }^{\circ} \mathrm{C}$ and $4000^{\prime} \mathrm{g}$. Then recombinant protein was extracted from the cells.

\section{Protein extraction and purification}


One hundred microliters of culture $\left(\mathrm{OD}_{600}=3\right)$ was collected by centrifugation $\left(4000^{\prime} \mathrm{g}, 5 \mathrm{~min}, 4^{\circ} \mathrm{C}\right)$ and resuspended in $400 \mathrm{~mL}$ lysis buffer $(50 \mathrm{mM}$ sodium phosphate, $300 \mathrm{mM} \mathrm{NaCl}, 0.01 \%$ Tween-20, $1 \mathrm{mM}$ PMSF, pH 8.0). Cell disruption was achieved by ultrasonic vibration with $20 \%$ power (SONIC uibra cell). The supernatant containing the recombinant protein was collected following centrifugation (4000' $\mathrm{g}, 5$ min, $4^{\circ} \mathrm{C}$ ). The target protein was isolated using an ÄKTA purifiler ${ }^{\mathrm{TM}}$ UPC10 system.

\section{M1/2/3 pull-down assay}

His-M1/2 and His-M3 fusion proteins were immobilized to beads (Dynabeads ${ }^{\mathrm{O}}$ His-Tag Isolation) and washed four times with washing buffer (50 mM sodium phosphate, $300 \mathrm{mM} \mathrm{NaCl}, 0.01 \%$ Tween-20, pH 8.0). A total protein (or purified Tkl1p-His protein) from a yeast culture in BMMY or BMGY medium was added (Reaction solution). The mixture was incubated for $30 \mathrm{~min}$ and the beads washed four times with A solution ( $3.25 \mathrm{mM}$ sodium phosphate, $70 \mathrm{mM} \mathrm{NaCl}, 0.01 \%$ Tween-20, $\mathrm{pH}$ 7.4) (Washing solution). Finally, $50 \mathrm{~mL}$ His elution buffer ( $300 \mathrm{mM}$ imidazole, $50 \mathrm{mM}$ sodium phosphate, $300 \mathrm{mM} \mathrm{NaCl}, 0.01 \%$ Tween-20, pH 7.4) was added to the samples and these were stirred for $2 \mathrm{~min}$. The His-tagged protein was eluted (Elution solution), and proteins that interacted with the His-tag protein were transferred to sample tubes. All mixtures were subjected to SDS-PAGE and MS analysis.

\section{M1/2 yeast two-hybrid}

The protocol from TAKARA was used. Y2HGold cells transformed with the M1 or M2-pGBKT7 recombinant plasmids (SD) were cultured in YPD medium. Y187 with the AD-pGADT7 recombinant plasmid $(A D)$ were also cultured in YPD medium. Equal volumes of the cultures containing SD and $A D$ were then mixed and added to $0.5 \mathrm{~mL} 2^{\prime}$ YPDA. The resuspended cells were mixed thoroughly. This mixture was incubated overnight $(20-24 \mathrm{~h})$ at $30^{\circ} \mathrm{C}, 200 \mathrm{rpm}$. Finally, a $100 \mathrm{~mL}$ aliquot of the incubated mating culture was incubated in SD/Try/Leu/His/Ade medium at $30^{\circ} \mathrm{C}$ for $3-5 \mathrm{~d}$ until visible single colonies appeared.

\section{Dmxr1 mutant construction}

The $P$. pastoris strain carrying the $m x r 1$ gene deletion was constructed by homologous recombination using kan as a marker. The upstream region of the $m x r 1$ gene was amplified initially by PCR using Pfu DNA polymerase (Thermo Scientific) and the P. pastoris genome as the template. The primers for this PCR, mxr1s-1 and mxr1s-2, included Sphl and BamHI restriction sites, respectively. The 0.6-kb PCRamplified fragment was inserted into Sphl/BamHI-digested pMD ${ }^{\text {TM }} 19-{ }^{-T}$ plasmid (TAKARA) to create the pMXR1UP plasmid. The downstream region of the mxr1 gene was also amplified with primers $m \times r 1 x-1$ and mxr1x-2, carrying restriction sites for $K p n l$ and $E c o R l$, respectively. This $0.5-\mathrm{kb}$ PCR fragment was inserted into pMD19-T to yield the pMXR1Down plasmid. Next, the G418 resistance gene with its own promoter and terminator (1556 bp) was amplified by PCR, using pFA6a-KanMX6 as the template and primers kan-1 and kan-2, which carried $B a m H I$ and $K p n l$ restriction sites, respectively, and the fragment was cloned into the Sphl/BamHI-digested plasmid pMXR1UP to give the pMXR1UP-Kan plasmid. This plasmid was digested with $\mathrm{Kpnl} / \mathrm{EcoRI}$ to generate a 2.2-kb fragment that was then inserted into 
Kpnl/EcoRI-digested pMXR1Down plasmid, yielding a P. pastoris $m \times r 1$ deletion plasmid, pMD19-T-MXR1del. The deletion cassette was released from pMD19-T-MXR1-del as a 2.7-kb EcoRI/Sphl-digested fragment and transformed by electroporation into wild-type $P$. pastoris (strain $X$-33). G418-resistant transformants were isolated on YPD supplemented with $1 \mathrm{mg} / \mathrm{mL} \mathrm{G} 418$. The correct integration of the deletion cassette into the genome and replacement of the $m x r 1$ open reading frame (ORF) in the transformants was confirmed by PCR analysis and Sanger sequencing.

\section{$m \times r 1$ and $t k l$ overexpression strain construction}

The $m x r 1$ gene and $t k /$ were amplified by PCR using genomic DNA as the template, and different primers. The fragment was ligated into the $\mathrm{pMD}^{\mathrm{TM}} 19-\mathrm{T}$ plasmid (TAKARA) and sequenced. The recombinant plasmid and pGAPZB plasmid (Invitrogen) were then digested with $P m \Lambda / X h o l$, and the $m x r 1$ fragment was inserted into pGAPZB to yield pGM. Finally, pGM was digested with Avrl and electro-transformed into the $P$. pastoris $X-33$ Dmxr1 mutant and P. pastoris X-33 wild-type, yielding Mxr1 overexpression strains $m x r 1-D m x r 1$ and $m x r 1-w t$, respectively. $t k / 1$ were amplified by PCR using genomic DNA as the template. Purified PCR products were $P m \Lambda / K p n l$-digested. This was followed by ligation into pGAPZB to create pGAPZB-tk/1. Then all the above plasmids were transformed into $X$-33 respectively by electrotransformation.

\section{Real-time PCR}

Total RNA extraction was carried out according to the standard procedure described in the Quantscrip TR kit (TAKARA). The reaction consisted of $5^{\prime}$ gDNA Eraser Buffer $(2 \mu \mathrm{L})$, gDNA Eraser $(1.0 \mu \mathrm{L})$, total RNA (4 $\mu \mathrm{L}, 500 \mathrm{ng})$ and $\mathrm{RNase}-F$ ree $\mathrm{ddH}_{2} \mathrm{O}(3 \mu \mathrm{L})$. Samples were incubated at $42{ }^{\circ} \mathrm{C}$ for $2 \mathrm{~min}$. cDNA served as the template for real-time PCR. The reverse transcription system contained $5 \mu \mathrm{L}$ of the SYBR Premix Ex Taq II (2'), both forward and reverse primers $(10 \mu \mathrm{M}, 0.5 \mu \mathrm{L})$, cDNA $(1 \mu \mathrm{L})$ and $\mathrm{dH}_{2} \mathrm{O}(3 \mathrm{~mL})$. Each experiment was carried out in triplicate. The transcription level was normalized to an endogenous reference gene, glyceraldehyde-3-phosphate dehydrogenase (gapdh). The data processing method was $2^{-\mathrm{DDCT}}$ [31].

\section{Western blotting detection}

Total protein samples were taken from cells cultured in YPD. Cultivating E. coli BL21 cells were transferred from $1 \times$ YPD after $16 \mathrm{~h}$ to $2 \times$ YPD. Cells were harvested $\sim 6 \mathrm{~h}$ later and the cell pellet washed twice with ice-cold $50 \mathrm{mM}$ potassium phosphate buffer ( $\mathrm{pH}$ 7.0). An electronic oscillator was used to disrupt cells to obtain soluble proteins. Before oscillation, $200 \mu \mathrm{L}$ of ice-cold PEBF $(0.7882 \% \mathrm{Tris}-\mathrm{HCl}$, $0.0585 \%$ EDTA and $2 \mu \mathrm{L}$ of $100 \mathrm{mM}$ PMSF) and glass beads were also added to ensure efficient cell disruption. The lysed cells were centrifuged at $10,000 \mathrm{~g}$ for $1 \mathrm{~min}$, and the supernatant was collected and used for SDS-PAGE analysis.

\section{Xylulose detection}


Wild-type $(X-33)$ cells and Tkl1p over-expression strain cells were harvested when the $\mathrm{OD}_{600}$ reached 6.0. The harvested cells were transferred from YPD to BMMY and incubated overnight at $30^{\circ} \mathrm{C}$ and 230 $\mathrm{rpm}$. Total protein extraction was the same as described above. The reaction system included formaldehyde (1-7\%), xylulose $(20 \mathrm{mM})$, and PEB and Mxr1 proteins $(25 \mathrm{~mL})$. The reaction was initiated by the addition of the crude cell extract $(70 \mathrm{~mL})$ and terminated with $20 \mathrm{~mL} \mathrm{H}_{2} \mathrm{SO}_{4}-\mathrm{Na}_{2} \mathrm{SO}_{3}(2 \mathrm{M}, \mathrm{pH} 2.0)$. High-performance liquid chromatography (HPLC) was performed to quantify the xylulose level. The HPLC parameters were as follows: an Aminexâ HPX-87H ion exclusion column, column temperature $60^{\circ} \mathrm{C}$, the flow rate of $0.6 \mathrm{~mL} / \mathrm{min}$ and $5 \mathrm{mM} \mathrm{H}_{2} \mathrm{SO}_{4}$ as eluent $[32,33]$.

\section{Formaldehyde and Methanol detection}

Cells were incubated overnight in BMGY medium ( $0.5 \%$ methanol) and then transformed into fresh BMMY medium incubate for $12 \mathrm{~h}, 30^{\circ} \mathrm{C}$. Cells were centrifuged and supernatant was collected for methanol and formaldehyde detection. HPLC was used for detection followed by Zhan[11]. Formaldehyde concentration was determined via Nash [34].

\section{Declarations}

\section{Funding}

This work was supported by the National Natural Science Foundation of China (21908077, 31570034), the national first-class discipline program of Light Industry Technology and Engineering (LITE2018-24), the Opening Project of the Key Laboratory of Industrial Biotechnology, Ministry of Education $\triangle$ KLIBKF201802】, the fifteenth batch of the "Six Talent Peaks Project in Jiangsu Province" (SWYY-180), the Collaborative Innovation Center of Jiangsu Modern Industrial Fermentation, the 111 Project (111-2-06).

\section{Author contributions}

Chunjun Zhan and Yankun Yang designed the research; Chunjun zhan and Yingyue Pan performed most of the experiments; Xiuxia Liu, Chunli Liu, Jinling Zhan, and Dinghua Xu analysed the data; Chunjun Zhan wrote the paper and Zhonghu Bai edited the manuscript.

\section{Corresponding author}

Crorespondence to Yankun Yang

\section{Ethical approval}

This article does not contain any studies with human participants performed by any of the authors.

\section{Consent for publication}

Not applicable. 
Conflict of interest

The authors declare that they have no conflict of interest.

\section{References}

1. Wu D, Zhu H, Chu J, Wu J: N-acetyltransferase co-expression increases alpha-glucosidase expression level in Pichia pastoris. Journal of biotechnology 2018.

2. Chen Q, Zhou Y, Yu J, Liu W, Li F, Xian M, Nian R, Song H, Feng D: An efficient constitutive expression system for Anti-CEACAM5 nanobody production in the yeast Pichia pastoris. Protein expression and purification 2018.

3. Yang Y, Fan FF, Zhuo R, Ma FY, Gong YM, Wan X, Jiang ML, Zhang XY: Expression of the Laccase Gene from a White Rot Fungus in Pichia pastoris Can Enhance the Resistance of This Yeast to H2O2Mediated Oxidative Stress by Stimulating the Glutathione-Based Antioxidative System. Applied and Environmental Microbiology 2012, 78:5845-5854.

4. Gassler T, Sauer M, Gasser B, Egermeier M, Troyer C, Causon T, Hann S, Mattanovich D, Steiger MG: The industrial yeast Pichia pastoris is converted from a heterotroph into an autotroph capable of growth on $\mathrm{CO}_{2}$. Nature Biotechnology 2019:1-7.

5. Cereghino JL, Cregg JM: Heterologous protein expression in the methylotrophic yeast Pichia pastoris. FEMS Microbiol Rev 2000, 24:45-66.

6. Johnson MA, Waterham HR, Ksheminska GP, Fayura LR, Cereghino JL, Stasyk OV, Veenhuis M, Kulachkovsky AR, Sibirny AA, Cregg JM: Positive selection of novel peroxisome biogenesis-defective mutants of the yeast Pichia pastoris. Genetics 1999, 151:1379-1391.

7. Kranthi BV, Kumar R, Kumar NV, Rao DN, Rangarajan PN: Identification of key DNA elements involved in promoter recognition by Mxr1p, a master regulator of methanol utilization pathway in Pichia pastoris. Biochimica et Biophysica Acta (BBA) - Gene Regulatory Mechanisms 2009, 1789:460-468.

8. Lin-Cereghino GP, Godfrey L, de la Cruz BJ, Johnson S, Khuongsathiene S, Tolstorukov I, Yan M, LinCereghino J, Veenhuis M, Subramani S, Cregg JM: Mxr1p, a Key Regulator of the Methanol Utilization Pathway and Peroxisomal Genes in Pichia pastoris. Molecular and Cellular Biology 2006, 26:883897.

9. Kranthi BV, Kumar HRV, Rangarajan PN: Identification of Mxr1p-binding sites in the promoters of genes encoding dihydroxyacetone synthase and peroxin 8 of the methylotrophic yeast Pichia pastoris. Yeast 2010, 27:705-711.

10. Zhan C, Yang Y, Zhang Z, Li X, Liu X, Bai Z: Transcription factor Mxr1 promotes the expression of Aox1 by repressing glycerol transporter 1 in Pichia pastoris. FEMS Yeast Research 2017, 17:fox015.

11. Zhan C, Wang S, Sun Y, Dai X, Liu X, Harvey L, McNeil B, Yang Y, Bai Z: The Pichia pastoris transmembrane protein $\mathrm{GT} 1$ is a glycerol transporter and relieves the repression of glycerol on AOX1 expression. FEMS Yeast Research 2016, 16:fow033. 
12. Parua PK, Ryan PM, Trang K, Young ET: Pichia pastoris 14-3-3 regulates transcriptional activity of the methanol inducible transcription factor Mxr1 by direct interaction. Molecular microbiology 2012, 85:282-298.

13. Parua PK, Ratnakumar S, Braun KA, Dombek KM, Arms E, Ryan PM, Young ET: 14-3-3 (Bmh) Proteins Inhibit Transcription Activation by Adr1 through Direct Binding to Its Regulatory Domain. Molecular and Cellular Biology 2010, 30:5273-5283.

14. Wang X, Wang Q, Wang J, Bai P, Shi L, Shen W, Zhou M, Zhou X, Zhang Y, Cai M: Mit1 Transcription Factor Mediates Methanol Signaling and Regulates the Alcohol Oxidase 1 (AOX1) Promoter in Pichia pastoris. The Journal of Biological Chemistry 2016, 291:6245-6261.

15. Wang X, Cai M, Shi L, Wang Q, Zhu J, Wang J, Zhou M, Zhou X, Zhang Y: PpNrg1 is a transcriptional repressor for glucose and glycerol repression of AOX1 promoter in methylotrophic yeast Pichia pastoris. Biotechnology Letters 2016, 38:291-298.

16. Sasano Y, Yurimoto $H$, Kuriyama M, Sakai Y: Trm2p-dependent derepression is essential for methanol-specific gene activation in the methylotrophic yeast Candida boidinii. Fems Yeast Research 2010, 10:535-544.

17. Sasano Y, Yurimoto H, Yanaka M, Sakai Y: Trm1p, a Zn(II)(2)Cys(6)-type transcription factor, is a master regulator of methanol-specific gene activation in the methylotrophic yeast Candida boidinii. Eukaryotic Cell 2008, 7:527-536.

18. Kumar NV, Rangarajan PN: The Zinc Finger Proteins Mxr1p and Repressor of Phosphoenolpyruvate Carboxykinase (ROP) Have the Same DNA Binding Specificity but Regulate Methanol Metabolism Antagonistically in Pichia pastoris. Journal of Biological Chemistry 2012, 287:34465-34473.

19. De Schutter K, Lin Y-C, Tiels P, Van Hecke A, Glinka S, Weber-Lehmann J, Rouzé P, Van de Peer Y, Callewaert N: Genome sequence of the recombinant protein production host Pichia pastoris. Nature biotechnology 2009, 27:561.

20. Yu H, Liao JC: A modified serine cycle in Escherichia coli coverts methanol and $\mathrm{CO}_{2}$ to two-carbon compounds. Nature Communications 2018, 9.

21. Woolston BM, King JR, Reiter M, Van Hove B, Stephanopoulos G: Improving formaldehyde consumption drives methanol assimilation in engineered E. coli. Nature communications 2018, 9:2387.

22. Witthoff S, Schmitz K, Niedenführ S, Nöh K, Noack S, Bott M, Marienhagen J: Metabolic engineering of Corynebacterium glutamicum for methanol metabolism. Appl Environ Microbiol 2015, 81:22152225.

23. Zhang W, Zhang T, Wu S, Wu M, Xin F, Dong W, Ma J, Zhang M, Jiang M: Guidance for engineering of synthetic methylotrophy based on methanol metabolism in methylotrophy. RSC Advances 2017, 7:4083-4091.

24. Savakis $\mathrm{P}$, Hellingwerf $\mathrm{KJ}$ : Engineering cyanobacteria for direct biofuel production from $\mathrm{CO}_{2}$. Curr Opin Biotechnol 2015, 33:8-14. 
25. Hu G, Li Y, Ye C, Liu L, Chen X: Engineering Microorganisms for Enhanced $\mathrm{CO}_{2}$ Sequestration. Trends in biotechnology 2018.

26. Gleizer S, Ben-Nissan R, Bar-On YM, Antonovsky N, Noor E, Zohar Y, Jona G, Krieger E, Shamshoum $\mathrm{M}$, Bar-Even A: Conversion of Escherichia coli to generate all biomass carbon from $\mathrm{CO}_{2}$. Cell 2019, 179:1255-1263. e1212.

27. Slekar KH, Kosman DJ, Culotta VC: The yeast copper/zinc superoxide dismutase and the pentose phosphate pathway play overlapping roles in oxidative stress protection. Journal of Biological Chemistry 1996, 271:28831-28836.

28. Krainer FW, Dietzsch C, Hajek T, Herwig C, Spadiut O, Glieder A: Recombinant protein expression in Pichia pastoris strains with an engineered methanol utilization pathway. Microbial cell factories 2012, 11:22.

29. Fukuoka H, Kawase T, Oku M, Yurimoto H, Sakai Y, Hayakawa T, Nakagawa T: Peroxisomal Fba2p and Tal2p complementally function in the rearrangement pathway for xylulose 5-phosphate in the methylotrophic yeast Pichia pastoris. Journal of bioscience and bioengineering 2019.

30. Singh K: Isolation of Multicopy Beta Lactamase Expression Strains of Pichia pastoris. 2007.

31. Livak KJ, Schmittgen TD: Analysis of relative gene expression data using real-time quantitative PCR and the 2(T)(-Delta Delta C) method. Methods 2001, 25:402-408.

32. Li H, Fan HC, Li YR, Shi GY, Ding ZY, Gu ZH, Zhang L: Construction and application of multi-host integrative vector system for xylose-fermenting yeast. Fems Yeast Research 2017, 17:fox055.

33. Sanchez-Moreno I, Garcia-Junceda E, Hermida C, Fernandez-Mayoralas A: Development of a new method for D-xylose detection and quantification in urine, based on the use of recombinant xylose dehydrogenase from Caulobacter crescentus. Journal of Biotechnology 2016, 234:50-57.

34. Nash T: The colorimetric estimation of formaldehyde by means of the Hantzsch reaction. Biochemical Journal 1953, 55:416.

\section{Tables}

Table 1 Strains used in this study 


\begin{tabular}{|c|c|c|}
\hline Strain & Genotype $^{\mathrm{R}}$ & $\begin{array}{l}\text { Source of } \\
\text { reference }\end{array}$ \\
\hline \multicolumn{3}{|l|}{ E.coli } \\
\hline Trans5 $\alpha$ & 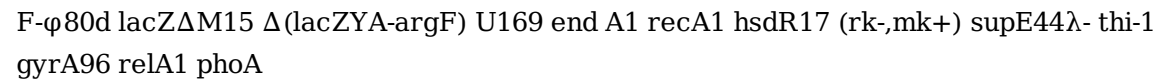 & TransGen Biotech \\
\hline Trans110 & $\begin{array}{l}\text { rpsL (StrR) thr leu thi-1 lacY gaIK gaIT ara tonA tsx dam dcm supE44 } \Delta \text { (lac-proAB) / } F^{\prime} \\
\text { [traD36 proAB lacIq lacZ } \Delta \mathrm{M} 15]\end{array}$ & TransGen Biotech \\
\hline$M \times r 1-B L 21(D E 3)$ & Recombinant plasmid (Mxr-pSVT7) transformed into $X-33$ & This study \\
\hline Pichia pastoris & & Invitrogen \\
\hline$x-33$ & Wide type $\mathrm{Mut}^{+}, \mathrm{His}^{+}$ & Invitrogen \\
\hline Dmxr1 & $X-33$ mxr1:: Kan & This study \\
\hline Mxr1-overexpression & Recombinant plasmid (Mxr-pGAPZB) transformed into $X-33$ & This study \\
\hline \multicolumn{3}{|l|}{ strain } \\
\hline$T K L 1-X-33$ & Recombinant plasmid (TKL1-pGAPZB) transformed into $X$-33 & This study \\
\hline Tkl-mxr1p double over & & This study \\
\hline \multicolumn{3}{|l|}{ expression } \\
\hline M2 & M2-pGAP transformed into Dmxr1 & This study \\
\hline M3 & M3-pGAP transformed into Dmxr1 & This study \\
\hline Saccharomyces cerevisias & & TAKARA \\
\hline \multirow[t]{3}{*}{ Y2HGold } & MATa, trp1-901, leu2-3, 112, ura3-52, his3-200, gal4 $\Delta$, gal80 $\Delta$, & TAKARA \\
\hline & LYS2 : : GAL1 UAS -Gal1 TATA -His3, GAL2 UAS -Gal2 TATA -Ade2 & \\
\hline & URA3 : : MEL1 UAS -Mel1 TATA AUR1-C MEL1 & \\
\hline Mxr1-1-Y2HGold & Recombinant plasmid (Mxr1-1- pGBKT7) transformed into Y187 & This study \\
\hline Mxr1-2-Y2HGold & Recombinant plasmid (Mxr1-2 pGBKT7) transformed into Y187 & This study \\
\hline \multirow[t]{2}{*}{ Y187 } & MATa, ura3-52, his3-200, ade2-101, trp1-901, leu2-3, 112, & TAKARA \\
\hline & gal4 $\Delta$, gal80 $\Delta$, met-,URA3 : : GAL1 UAS -Gal1 TATA -LacZ,MEL1 & \\
\hline TKL1-Y187 & Recombinant plasmid (DAS1- pGADT7)transformed into Y187 & This study \\
\hline IMD-Y187 & Recombinant plasmid (IMD- pGADT7) transformed into Y187 & This study \\
\hline$U D P-187$ & Recombinant plasmid (UDP- pGADT7) transformed into Y187 & This study \\
\hline
\end{tabular}

Zeocin $^{\mathrm{R},}$ Zeocin resistance

Table 2 Primers used in this study

\begin{tabular}{ll}
\hline Primer & Sequence \\
\hline mxr150-F(M1-F) & CC CATATG ATGAGCAATCTACCCCCAAC \\
mxr150-R(M1-R) & CC GGATCC TCA GTGGTGGTGGTGGTGGTG ATTTGAGTCCCGGCGGCC \\
mxr-400-F(M2-F) & GGGAATTC CATATG AGCAACTTACCGCCTACCTTTG \\
mxr-400-R(M2-R) & CGC GGATCC TTACGGTGCGGTATCTTTCGGC \\
mxr400B-F & CG GGATCC ATGAGCAACTTACCGCCTACCTTTG \\
mxr400B-R & CCCTCGAGTTA ATGATGGTGATGATGGTGGCAGC \\
Mxr1-1-F & ATGAGCAATCTACCCCCAAC \\
Mxr1-1-R & TCA GTGGTGGTGGTGGTGGTG ATTTGAGTCCCGGCGGCC \\
Mxr1-2-F & ATGAGCAACTTACCGCCTACCTTTG \\
Mxr1-2-R & TTACGGTGCGGTATCTTTCGGC \\
G1-F & TCAGAAAAGAACACCCTTCGTACTTG ATGAGCGCCTATCAATCTCCTA \\
G1-R & TCAGAAAAGAACACCCTTCGTACTTG CTAATGCTCCAAAATAGTAAGGTTACCTGT \\
G2-F & TCAGAAAAGAACACCCTTCGTACTTG ATGGCTAGAATTCCAAAAGCAGT \\
G2-R & TCAGAAAAGAACACCCTTCGTACTTG TTACAACTTGTCATGCTTTGGTTTC \\
G3-F & TCAGAAAAGAACACCCTTCGTACTTG ATGACAGGAGAACATAAACGAAGTTC \\
G3-R & TCAGAAAAGAACACCCTTCGTACTTG TCAGAAAAGAACACCCTTCGTACTTG \\
\hline
\end{tabular}




\section{Table 3 Plasmids used in this study}

\begin{tabular}{|c|c|c|}
\hline Plasmid & Genotypea & $\begin{array}{l}\text { Source or } \\
\text { reference }\end{array}$ \\
\hline pMD19-T & Ampicillin $^{\mathrm{R}}$; E. coli subcloning vector & This study \\
\hline \multicolumn{3}{|l|}{ Simple } \\
\hline pGBKT7 & Kanamycin $^{R}$; PTRP,1 C-Myc epitope tag, GAL4(1-147) -based expression vector & TAKARA \\
\hline $\begin{array}{l}\text { mxr1-1- } \\
\text { pGBKT7 }\end{array}$ & $\begin{array}{l}\text { Kanamycin }{ }^{R} \text {; PTRP, } 1 \text { C-Myc epitope tag, GAL4(1-147) -based expression vector PGAP-based expression } \\
\text { vector containing M1 gene }\end{array}$ & This study \\
\hline $\begin{array}{l}\text { mxr1-2- } \\
\text { pGBKT7 }\end{array}$ & $\begin{array}{l}\text { Kanamycin }{ }^{R} \text {; PTRP, } 1 \text { C-Myc epitope tag, GAL4(1-147) -based expression vector PGAP-based expression } \\
\text { vector containing M2 gene }\end{array}$ & This study \\
\hline $\begin{array}{l}\text { mxr1-3- } \\
\text { pGBKT7 }\end{array}$ & $\begin{array}{l}\text { Kanamycin }{ }^{R} \text {; PRR, } 1 \text { C-Myc epitope tag, GAL4(1-147) -based expression vector PGAP-based expression } \\
\text { vector containing mxr150-400 gene }\end{array}$ & This study \\
\hline pGADT7 & Ampicillin ${ }^{R}$; PTRP,1 HA epitope tag, GAL4(768-881) -based expression vector & TAKARA \\
\hline $\begin{array}{l}\text { DAS1- } \\
\text { pGADT7 }\end{array}$ & $\begin{array}{l}\text { Ampicillin }{ }^{R} \text {; PTRP, } 1 \text { HA epitope tag, GAL4(1-147) -based expression vector PGAP-based expression } \\
\text { vector containing DAS1 gene }\end{array}$ & This study \\
\hline pGADT7-M2 & $\begin{array}{l}\text { Ampicillin }{ }^{\mathrm{R}} \text {; PTRP, } 1 \text { HA epitope tag, GAL4(1-147) -based expression vector PGAP-based expression } \\
\text { vector containing IMD gene }\end{array}$ & This study \\
\hline pGADT7-M3 & $\begin{array}{l}\text { Ampicillin }{ }^{\mathrm{R}} \text {; PTRP,1 HA epitope tag, GAL4(1-147) -based expression vector PGAP-based expression } \\
\text { vector containing UDP gene }\end{array}$ & This study \\
\hline M2-pGAPZB & Zeocin ${ }^{\mathrm{R}} ; \mathrm{P}_{\mathrm{GAP}}-\mathrm{M} 2$, -based expression vector $\mathrm{P}_{\mathrm{GAPZB}}$-based expression vector & This study \\
\hline M3-pGAPZB & Zeocin ${ }^{\mathrm{R}} ; \mathrm{P}_{\mathrm{GAP}}-\mathrm{M} 3$, -based expression vector $\mathrm{P}_{\mathrm{GAPZB}}$-based expression vector & This study \\
\hline
\end{tabular}

\section{Figures}




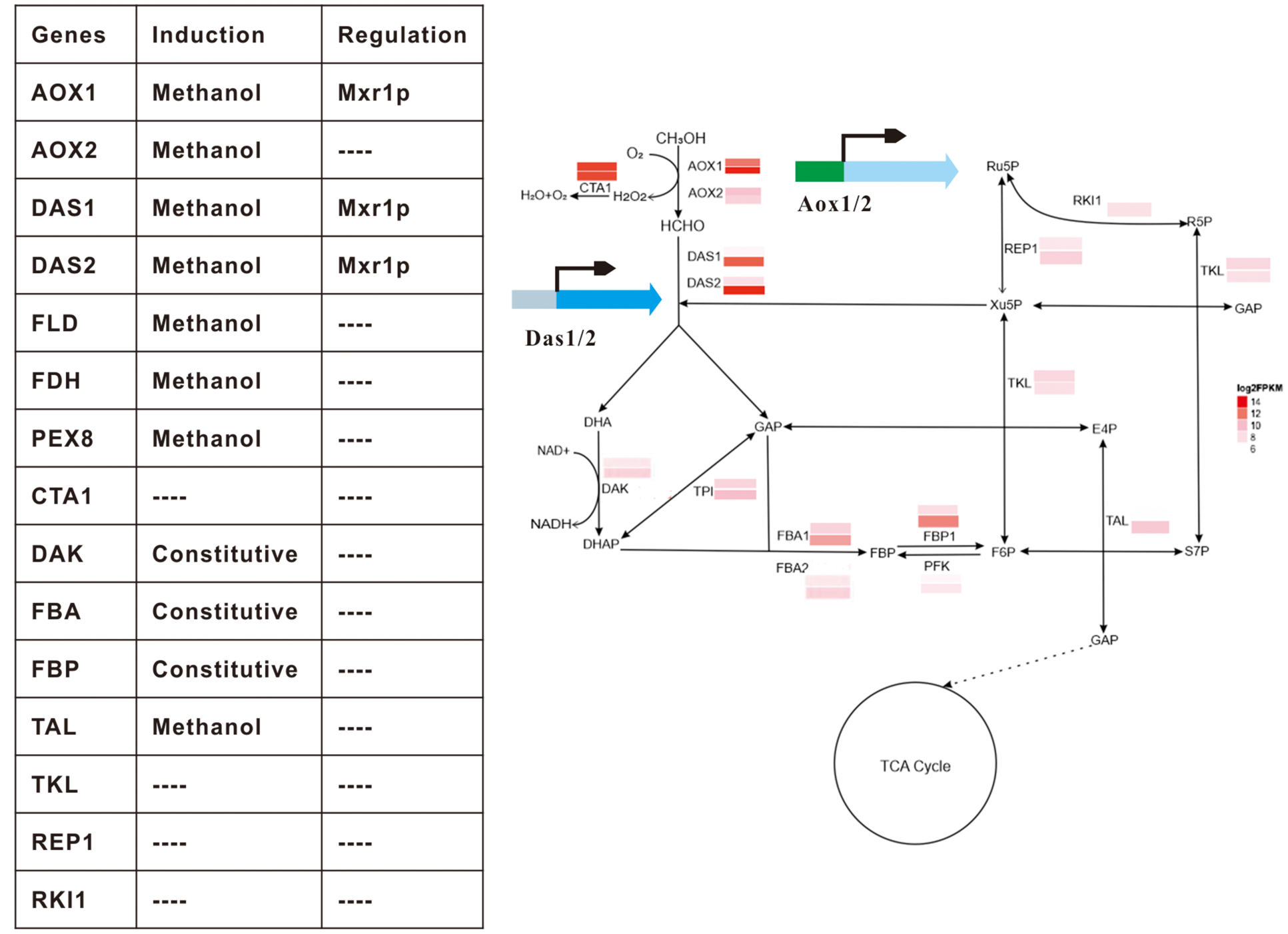

Figure 1

Schematic diagram of important genes related to methanol metabolic pathway (assimilation pathway). AOX1/2: alcohol oxidase1/2. DAS1/2: CTA1: catalase. DAK: dihydroxyacetone kinase. TPI:

triosephosphate isomerase. FBA1/2: fructose-1,6-bisphosphate aldolase1/2. FBP: fructose-1,6bisphosphate. TKL: transketolase. RPE1: ribulose-phosphate 3-epimerase. RPIA1: ribose 5-phosphate. TAL: transldolase. DHA: dihydroxyacetone. DHAP: dihydroxyacetone phosphate. GAP: glyceraldehyde-3phosphate. FBP: fructose-1,6-bisphosphate. F6P: fructose-6-phosphate. E4P: S7P: sedoheptulose-7phosphate. Xu5P: xylulose-5-phosphate. Ru5P: ribulose-5-phosphate. R5P: ribose-5-phosphate. 


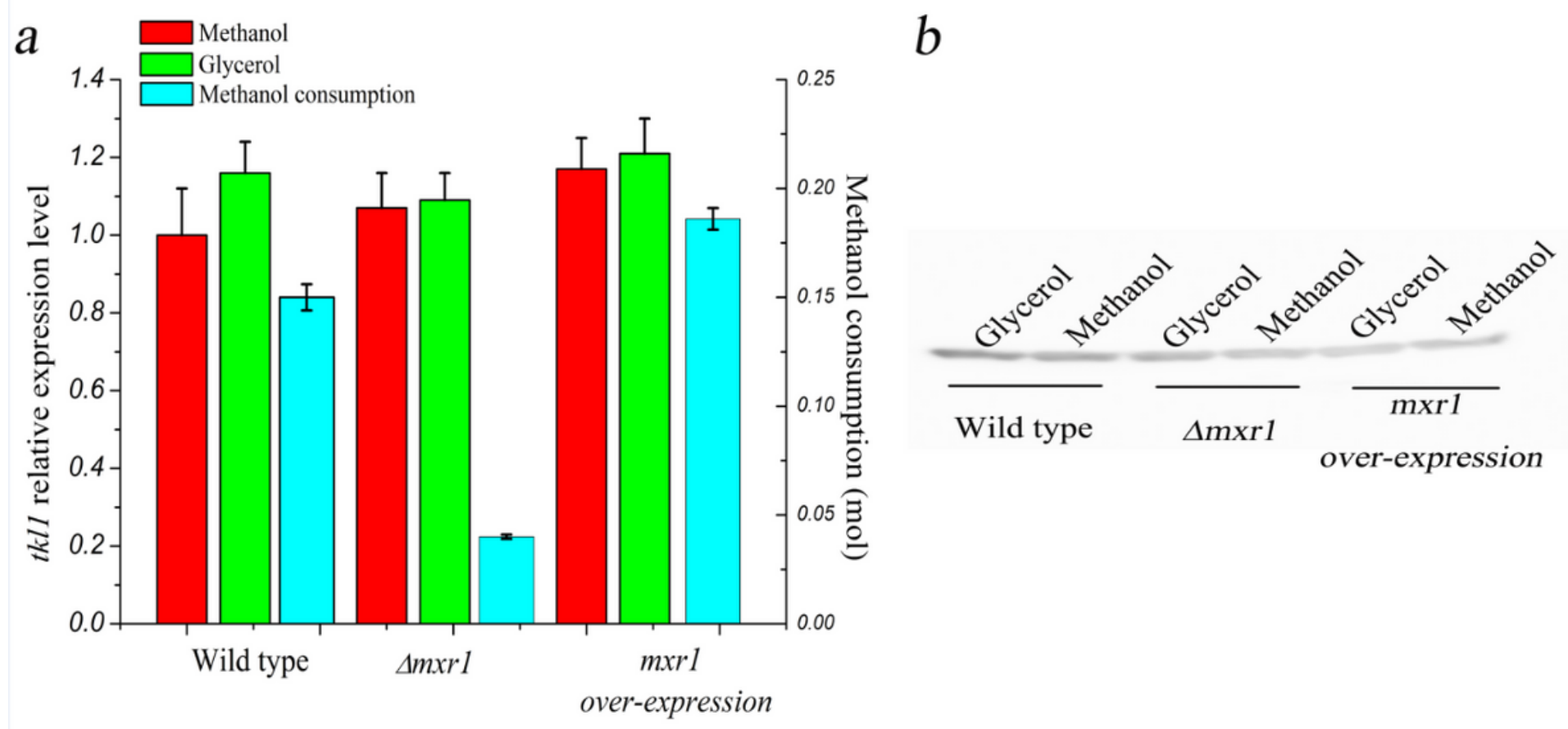

\section{Figure 2}

tkl1 expression levels in different conditions. (a) tkl1 transcriptional level and methanol consumption in different strains. (b) tkl1 translational level in different cells. 

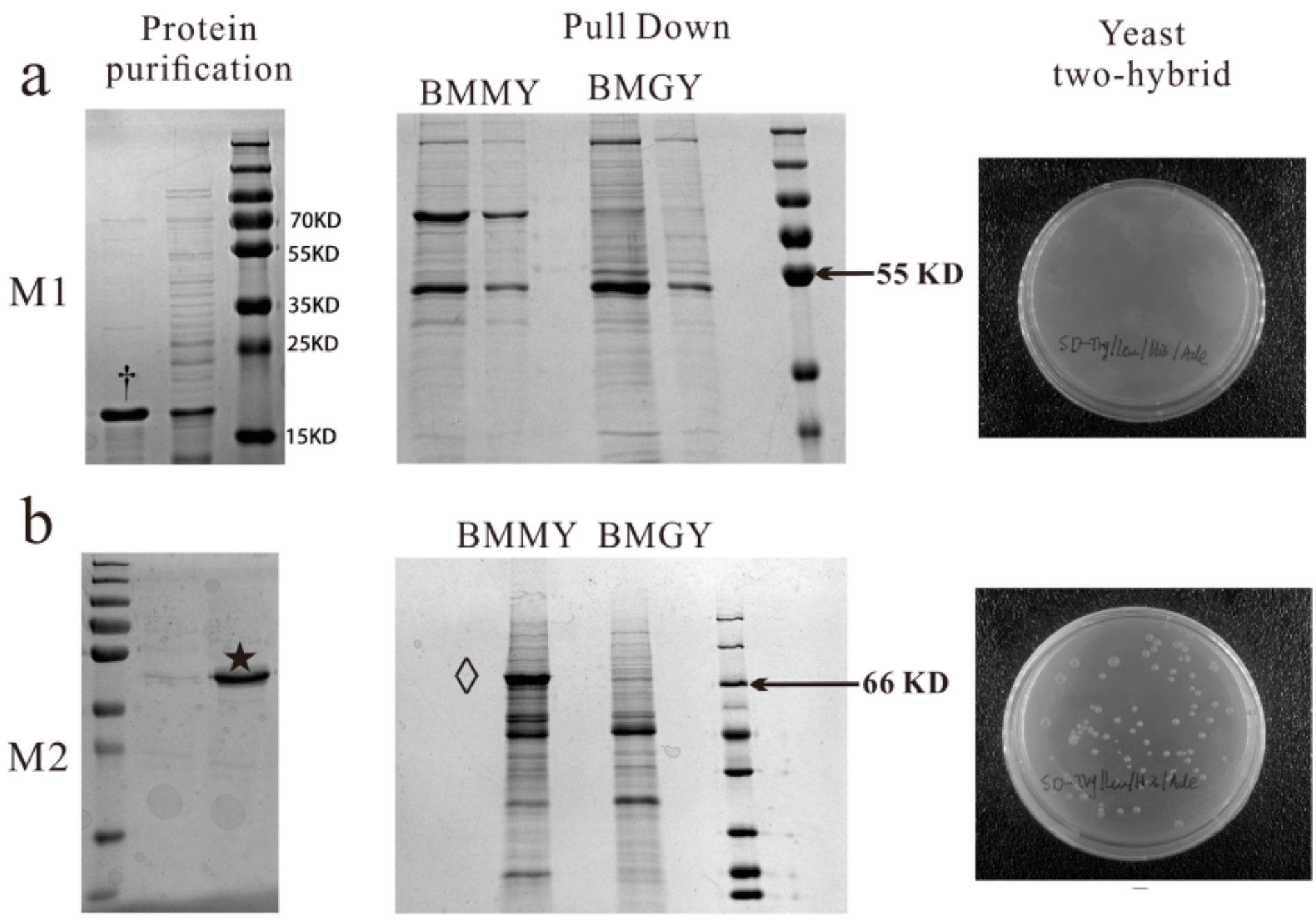

\section{Figure 3}

Mxr1p interacting protein diggnation. (a) Proteins interacted with M1 (Mxr1p-150AA). (b) Proteins interacted with M2 (Mxr1p-400AA). BMMY: Total proteins using for pull-down comes from BMMY medium. Total proteins using for pull-down comes from BMGY medium. †:M1. : M2. : Targeted band. 


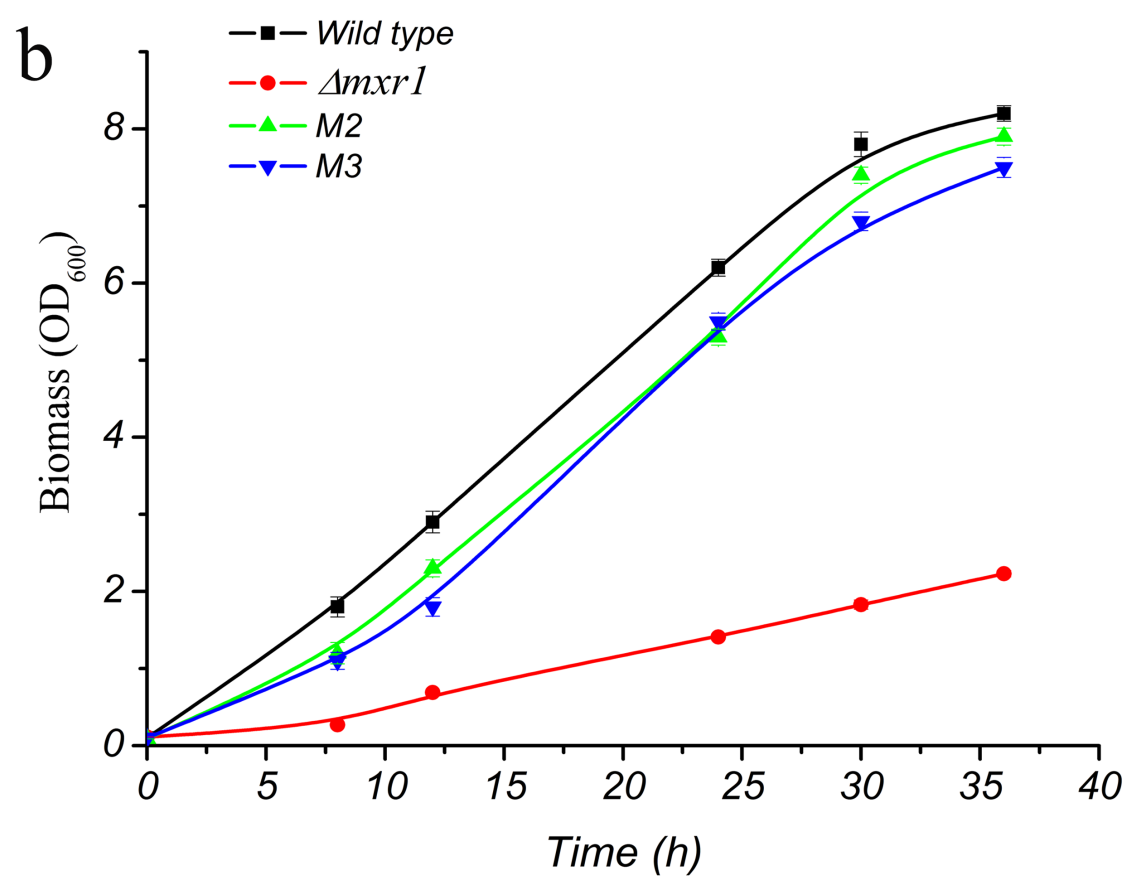

\section{Figure 4}

Interaction site studies between Mxr1p mutant and Tkl1. (a) pull-down assay using Tkl1-His and M3-Flag purified protein. (b) Different strains growth curve in methanol. $\square m \times r 1$ : mxr1 deletion. M2: integrate M2 into $\square m \times r 1$ mutant. M3: integrate M3 into $\square m \times r 1$ mutant.
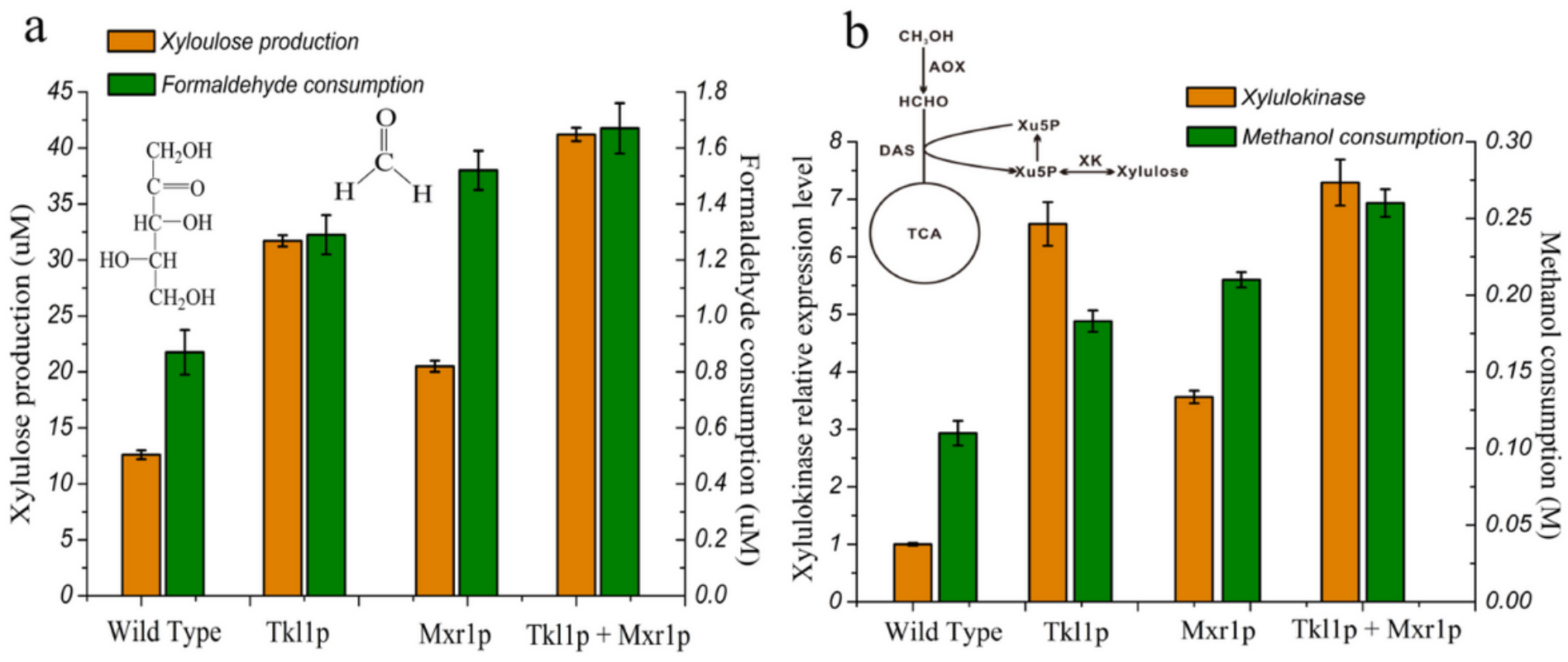

Figure 5

Evaluation Mxr1p-Tkl1p complex function. (a) Xylulose production and formaldehyde consumption in different reaction system. (b) Measurement of XK transcriptional level and methanol consumption in different reaction system 


\section{Supplementary Files}

This is a list of supplementary files associated with this preprint. Click to download.

- Abstract2.tif

- Supplement.docx 